\title{
คteknokultura
}

\#Puyosa, I.. (2015). Control político de internet en el contexto de un régimen híbrido Venezuela 2007-2015. Revista Teknokultura, Vol. 12(3), 501-526.

Recibido: $01-10-2015$

Aceptado: $02-12-2015$
Open peer review

http://revistas.ucm.es/index.php/TEKN/pages/view/opr-50392

\section{Control político de internet en el contexto de un régimen híbrido Venezuela 2007-2015}

\section{Political control on the Internet} in the context of a hybrid regime. Venezuela 2007-2015

Iria Puyosa

Universidad de las Américas

iria.puyosa@udla. edu.ec

\section{Resumen}

En este artículo se presenta un estudio de caso sobre las políticas de control, en el campo de internet y de la web social, desarrolladas por el Estado venezolano, entre $2007 \mathrm{y}$ 2015. El estudio de caso incluye examen de documentos fundamentales de la política pública para internet del Estado venezolano, estadísticas del sector y datos aportados por investigaciones previas, y sistematización de eventos empíricos que dan cuenta de las características de las prácticas de control político de la web que fueron instrumentadas por el Estado venezolano. El estudio analiza si los datos permiten sustentar las hipótesis del "dilema del dictador" (Kerr, 2014) en un régimen híbrido como el venezolano. 
estudio analiza si los datos permiten sustentar las hipótesis del "dilema del dictador" (Kerr, 2014) en un régimen híbrido como el venezolano.

\section{Palabras Clave}

Control comunicacional; "dilema del dictador"; denegación de acceso; vigilancia gubernamental; disputa contenciosa en la web.

\section{Abstract}

This article presents a case study on control policies on the field of the internet and the social web, developed by the Venezuelan government, between 2007 and 2015. The case study includes examination of key documents of public policy for internet by Venezuelan State, industry statistics and data provided by previous research, as well as systematization of empirical events that reflect the characteristics of the practices of political control on the web that were implemented by the Venezuelan State. The study examines whether the data substantiate the hypotheses of the "dictator's dilemma" (Kerr, 2014) in a hybrid political regime as Venezuela.

\section{KeYWORDS}

Communication control; "Dictator's dilemma"; denial of access; government surveillance; contentious dispute on the web.

\section{SUMARIO}

Introducción: El régimen venezolano y el cambio en su política pública para internet El dilema del dictador digital y el tablero de juego de Venezuela 2001-2007: Democracia Delegativa e Internet Libre 2007-2013: Emergen los controles de segunda generación 2013-2015: El control de internet en un régimen híbrido con tendencias autocráticas Conclusiones

Referencias 


\section{Summary}

Introduction: The Venezuelan regime and the change in its public policy for the Internet Digital dictator 's dilemma and the Venezuelan game board 2001-2007: Delegative Democracy and Free Internet 2007-2013: Rising of second generation controls 2013-2015: Internet control in a hybrid regime with autocratic tendencies Conclusions

References 



\section{Introducción:}

\section{El régimen venezolano y el cambio en su política pública para internet}

A partir de 2007 ocurre un viraje en las políticas públicas del gobierno de Venezuela en rela ción con internet. Coinciden Briceño (2009), Benítez \& Pietrosemoli (2012) y Urribarri (2013) en destacar que los cambios en los lineamientos generales de las políticas públicas para internet, refleja un cambio de modelo. En contraposición al modelo de apertura que había es tado vigente al inicio del siglo, luego de 2007 comienza a establecerse un modelo de control político, que coincide con el proyecto general de hegemonía comunicacional (Bisbal, Oropeza, Hernández, Urribarri, Cañizalez, Correa, Abreu \& Quiñonez, 2009).

De acuerdo con las proposiciones del Marco de Coaliciones de Causa (Sabatier, 1993; Sabatier \& Jenkins-Smith, 1993; Sabatier \& Weible, 2007; Weible \& Nohrstedt, 2012), significativas perturbaciones externas al sub-sistema de política pública, facilitan que se produzca un cambio en los atributos centrales de una política pública. El estudio de los sustanciales cambios en la orientación de la política pública del Estado venezolano para internet entre 1999-2006 y 2007-2015, es interesante dado que la misma élite política se mantuvo en el poder entre los dos períodos. El Marco de Coaliciones de Causa propone las hipótesis de cambios en el sistema de creencias y en las oportunidades políticas, en un contexto en el cual el mismo partido político se ha mantenido en el poder (Sabatier \& Weible, 2007; Weible \& Nohrstedt, 2012).

En este estudio, plantea la transición de un régimen que ha sido catalogado como democracia delegativa (Chaguaceda \& Puerta, 2015), autoritarismo competitivo o autoritarismo electoral (Alvarez, 2011), a un régimen híbrido crecientemente autocrático (Corrales \& Hi dalgo, 2013).

A partir de 2007, el régimen político imperante en Venezuela muestra una tendencia al in cremento de prácticas autoritarias. Corrales \& Hidalgo (2013) señalan como elementos de este proceso de creciente autoritarismo: la delegación del poder de la Asamblea Nacional en el Presidente de la República; la aprobación por decreto de leyes que coliden con la Constitución de la República; la sistemática inhabilitación política de dirigentes opositores; la eliminación de competencias y facultades que anteriormente tenían gobernadores y alcaldes; la reelección presidencial indefinida; la expansión a internet ("medios electrónicos") de las disposiciones de control de contenidos de la Ley de Responsabilidad Social en Radio y Televisión; la revoca- 
toria de concesiones a estaciones de radio y televisión opositoras o críticas al gobierno; el establecimiento del modelo político del "poder popular" basado en órganos comunales que reportan directamente a la Presidencia de la República; la prohibición de financiamiento externo para organizaciones de defensa de derechos humanos; el monopolio de operaciones de cambio de divisas por parte del gobierno nacional; el establecimiento de facto del criterio de lealtad política al partido de gobierno como requisito para el nombramiento como juez; la reforma electoral de 2010 que cambió a un sistema mayoritario que permitió la sobrerrepresentación de legislativa del partido de gobierno; el uso de los recursos del Estado en las campañas electorales del partido PSUV; el amedrentamiento directo a los votantes que deben asistir a los centros de votación acompañados por activistas locales del oficialismo, etc.

En este contexto, el sistema de ideas que sustentaba la política de apertura de telecomunicaciones y ampliación del acceso a internet como fundamentos de un proceso de desarrollo económico y democratización de la sociedad, pareciera haber entrado en conflicto con los intereses de la élite en el poder. La evidencia indica que el incremento de los debates críticos al gobierno chavista y al proceso bolivariano en el espacio de contestación de la web a partir de 2009 fueron condicionantes en la valoración de las opciones de democratización o de control en el campo de internet por parte de los responsables de las políticas públicas para internet en Venezuela.

\section{El dilema del dictador digital y el tablero de juego de Venezuela}

A medida que ha ido aumentando la penetración de internet y se ha hecho evidente la cre ciente importancia de sus usos políticos, regímenes de todo tipo (democráticos, híbridos y autocráticos) han establecido políticas de creciente control y securitización de internet, tal como reflejan los informes de Freedom on the Net (Freedom House, 2015). Las prácticas más evidentes han sido de filtrado de palabras clave y bloqueo de contenidos, mas a partir de 2009 empiezan a usarse los controles de segunda-generación: corte total de acceso a internet en re giones en donde se producen protestas, bloqueo just-on-time de páginas web o aplicaciones móviles, requerimiento informales de remoción de contenidos a ISPs y a administradores de sitios web, ralentización de las conexiones a internet en momentos de alta conflictividad política y establecimiento de altos costos de los servicios para asegurar las limitaciones al acceso 
(Deibert \& Rohozinski, 2010; Howard, Agarwal \& Hussain, 2011; Pearce \& Kendzior, 2012; Crete-Nishihata, Deibert \& Senft, 2013; Murdoch \& Roberts, 2013).

También se ha encontrado en la literatura evidencia de que los países con regímenes auto ritarios tienden a limitar el desarrollo de la infraestructura de acceso a internet y a incrementar los controles sobre los proveedores de servicios (Drezner, 2009). Asimismo, se encuentra evidencia de prácticas de control y securitización que no requieren mecanismos técnicos sino que se basan en mecanismos policiales, judiciales o administrativos: impuestos al uso de internet, requerimientos administrativos a los proveedores de acceso, reportes policiales rutinarios de la actividad ciudadana en internet y prisión por expresión política en la web (Drezner, 2009; Kerr, 2014). Finalmente, se observan prácticas de monitoreo masivo (en ocasiones visibilizado por el propio gobierno), uso de información publicada en-línea para hostigar o acusar legalmente a activistas políticos, grupos de "ciberactivistas" pagados para in terferir en debates sobre asuntos políticos y ciberataques perpetrados por crackers contra activistas opositores o "patriotic hacking" (Deibert \& Rohozinski, 2010; Drezner 2010; Morozov, 2012; Kerr, 2014).

Las proposiciones del "dilema del dictador" (Kerr, 2014) indican que el establecimiento de medidas de control de internet depende de un proceso de toma de decisiones en el cual se consideran factores internos y externos. El dilema consiste es establecer un balance entre la reputación internacional, el valor de los flujos económicos afectados por el uso de internet y el control político de la expresión del descontento y la organización de protestas. Los factores in ternos incluyen aumento de los usos políticos de internet, nivel de protestas, importancia para la economía nacional del acceso a internet, capacidad del Estado para establecer restricciones; mientras que los factores externos incluyen costos de reputación internacional, vulnerabilidad del país a la presión internacional y establecimiento de políticas restrictivas por países aliados (Kerr, 2014).

Considerando estas variables, el modelo del "dilema del dictador" hipotetiza que en regímenes híbridos, los mecanismos tradicionales de filtrado de palabras clave y bloqueo de contenidos son poco sofisticados y selectivos, aplicándose sólo en casos específicos para los cuales pueden legitimarse (ej. terrorismo, pornografía infantil); dichas restricciones podrían ampliarse en situaciones de protestas masivas, especialmente si dichas protestas son amplificadas por el uso de la web. El modelo también hipotetiza que el uso de mecanismos de control de segunda generación se hace más probable y aumenta en sofisticación en correlación con el aumento de la penetración de internet y sus usos políticos. 
En caso de regímenes híbridos, catalogados como autoritarismos competitivos o regímenes ya autocráticos que intentan mantener reputación internacional de cierto grado de democracia (como es el caso de Venezuela), el incremento de la penetración de internet y sus usos políticos actúa como un disparador del establecimiento de políticas de control de segunda generación. Los mecanismos de control de primera generación, filtrado de palabras clave y bloqueo de contenidos, son considerados muy costosos en términos de reputación internacional y legitimidad interna, por lo tanto sólo son utilizados por este tipo de regímenes bajo la presión de movimientos masivos de protesta que amenacen su estabilidad. Es decir, que en un país de régimen híbrido como Venezuela, primero aparecerían formas sofisticadas y selectivas de control político de internet de segunda generación y sólo en momentos de alta conflicti vidad política se usarían mecanismos de control de primera generación.

\section{1-2007:}

\section{Fase de Democracia Delegativa e Internet Libre}

Entre 2001 y 2009, el régimen de Venezuela fue clasificado en los estudios políticos como una democracia delegativa (Chaguaceda \& Puerta, 2015) o un autoritarismo competitivo (Alvarez, 2011). Entre las características que O’Donnell (2001) atribuye a la democracia delegativa se aplican al régimen venezolano (2001- 2007) las siguientes: a) Tiene legitimidad de origen; b) Tienen el respaldo de resultados electorales; c) El ejercicio del poder entra en conflicto con la institucionalidad política; d) El ejercicio del poder intenta neutralizar a las instituciones; e) Se centraliza en el Ejecutivo y se tiende al personalismo; f) El "líder" está por encima de las instituciones; g) El "líder" se presenta como "salvador de la Patria"; h) Tiene apoyo en sectores significativos de la sociedad.

En la fase de democracia delegativa, las políticas públicas para internet del Estado venezolano fueron afines a las esperables en un régimen democrático en un país en vías de desarrollo. En los primeros años del siglo, la colaboración entre el Consejo Nacional de Tecnologías de la Información (CNTI), redes académicas y el empresariado afiliado a CAVECOM-E facilitó la modernización del marco regulatorio para el sector, incluyendo la Ley de Telecomunicaciones (2000). En esa etapa, CONATEL formula y aprueba el Plan Nacional de Telecomunicaciones que tenía entre sus objetivos: 
insertar a la Nación dentro del concepto de sociedad del conocimiento (...) teniendo en cuenta que, para el desarrollo de estos procesos, la red mundial denominada internet, representa en la actualidad y en los años por venir, un medio para la interrelación con el resto de los países y una herramienta invalorable para el acceso y difusión de ideas.

(CONATEL, 2000).

Para el lanzamiento de Venezuela hacia la sociedad del conocimiento fue fundamental el Decreto 825. En el artículo 1 de ese decreto se declaraba “...el acceso y el uso de Internet como política prioritaria para el desarrollo cultural, económico, social y político de la República Bolivariana de Venezuela" (Presidencia de la República Bolivariana de Venezuela, 2000). En el año 2001 se aprueba la Ley Orgánica de Ciencia, Tecnología e Innovación (LOCTI) que fo mentaba el uso de recursos privados para el desarrollo en ciencia y tecnología. De esa gestión también salió el proyecto Infocentros, que proponía la creación de centros comunitarios para masificar el acceso a internet y dar formación en uso de TICs a los sectores populares. En 2004, se decreta el uso preferente del software libre en la administración pública (Decreto 3.390), que es el antecedente del desarrollo del sistema operativo Canaima (distribución GNU/Linux venezolana basada en Debian) y del proyecto educativo Canaimitas. Estas políticas públicas, apoyadas por el trabajo mancomunado del Estado, las universidades y las empresas privadas del sector, llevaron a que la penetración de internet pasara de 3\% (2000) a 21\% (2007) (Puyosa, 2015a).

En el período de 2002 a 2007, la web se constituyó en un espacio para la circulación de información y para expresión política en Venezuela (Briceño, Núñez, Pisanty, Puyosa, Urribarrí \& Torrens, 2010; Puyosa, 2012; 2015a). Este espacio facilitó la incorporación de nuevos actores y nuevas voces al diálogo colectivo (Urribarri, 2011; Delgado \& Arenas, 2014) y proporcionó opciones para la expresión contra-hegemónica (Delgado \& Puyosa, 2011; Puyosa, 2015b). La disputa política en los espacios de la web comienza prácticamente desde la llegada al poder del chavismo, en paralelo al proceso de polarización política. En 2002, se funda Aporrea.org, foro de discusión de activistas del chavismo, que durante 13 años ha sido uno de los espacios más importantes para la conversación política venezolana en la web (Puyosa, 2015a). A partir de 2004, la blogosfera venezolana también pasó a ser un espacio de confrontación ideológica, sin mayores interferencias del Estado en la libre circulación de información y en la expresión política, al margen de las políticas de hegemonía comunicacional que se aplicaban en los medios masivos (Bisbal et al, 2009; Cañizalez, 2012). 


\section{7-2013:}

\section{Emergen los controles de segunda generación.}

A partir de 2007, el régimen político venezolano comienza a acentuar sus rasgos autoritarios (Alvarez, 2011; Corrales \& Hidalgo, 2013; Chaguaceda \& Puerta, 2015). Gradualmente, se empiezan a observar cambios en las políticas públicas para internet. Ya en 2010, resulta claro el cambio de un modelo democratizador del acceso a las tecnologías de información y comunicación a un modelo de control político con pretensiones hegemónicas (Urribarri, 2013; Puyosa, 2015a). El hito inicial es el Plan Nacional de Telecomunicaciones 2007-2011, que se alinea con el Proyecto Nacional Simón Bolívar, Primer Plan Socialista de la Nación 20072013, específicamente con el objetivo de "Consolidar el sistema de comunicación nacional como instrumento para el fortalecimiento de la democracia protagónica revolucionaria" (Presidencia de la República Bolivariana de Venezuela, 2007). En mayo de ese mismo año, se reestatiza la compañía telefónica CANTV y se termina con el proceso de apertura de inversiones en el sector telecomunicaciones iniciado 6 años antes.

Las consecuencias de la re-estatización de CANTV se comenzarían a observar con la desinversión en infraestructura de telecomunicaciones que se registra a partir de 2008, de acuerdo con los informes de presupuesto de la propia empresa (Puyosa, 2015a). Las inversiones públicas en el sector telecomunicaciones cayeron, entre 2007 y 2009, incluso en términos de Bs. nominales, sin ajuste por inflación. ${ }^{1}$ Asimismo, entre 2009 y 2014, los organismos a cargo de administrar el control de cambios — primero CADIVI y luego CENCOEX - restringieron la asignación de divisas para que los proveedores privados de internet impor taran tecnología y equipos para la infraestructura de conectividad (Puyosa, 2015a). Este proceso de limitación del desarrollo de infraestructura de acceso a internet puede ser interpretado como una política de denegación de acceso de segunda generación (Drezner, 2009).

En 2008, se registró un intenso debate sobre el establecimiento de un punto único de conexión a internet para todo el país, que sería controlado por el gobierno nacional. Aunque nunca se conoció una propuesta oficial detallada, su mención en el borrador del Proyecto de Ley Orgánica de Telecomunicaciones, Informática y Servicios Postales que circuló ese año en-

Según los informes anuales de CANTV, de una inversión de Bs. 3.620.859.602 en 2007 se cae a Bs. 3.041.653.387 en 2008 y Bs. 3.391.978.528 en 2009 (con una inflación acumulada de 54\% entre los 2 años). 
cendió alarmas sobre el interés del Estado venezolano en controlar el acceso a internet (Urribarri, 2012). El punto único de acceso a internet habría facilitado al Estado venezolano el establecimiento de un sistema de control de internet de primera generación, es decir centrado en el bloqueo de dominios y direcciones así como en el filtrado por palabras clave. Dado que en ese momento, el gobierno chavista todavía mantenía reconocimiento como un régimen con bases democráticas, el uso de prácticas de control de internet de primera generación no resultaba conveniente para sostener su reputación internacional y su legitimidad interna. Sumado al hecho de que la penetración de internet en Venezuela era de apenas 23\% (CONATEL, 2013), no es de extrañar que la política haya sido desechada y se haya preferido explorar controles de segunda generación que tenían menor costo político.

El punto de quiebre en la política del Estado venezolano para internet es el Decreto 6.649 o Instructivo Presidencial para la Eliminación del Gasto Suntuario o Superfluo en el Sector Público Nacional (Presidencia de la República Bolivariana de Venezuela, 2009). Al calificar de suntuario el uso de internet en la administración pública y en las instituciones del Estado, el decreto 6.649 contravenía la condición prioritaria conferida en el decreto 825 (2000). La restricción de las inversiones en internet en las universidades nacionales es un paso importante en el establecimiento de políticas de denegación de acceso de segunda generación, en este caso basada en mecanismos administrativos e impositivos (Drezner, 2009; Kerr, 2014).

En respuesta al decreto 6.649, surgió el movimiento Internet Prioritaria que se planteó como objetivo lograr que el Ejecutivo Nacional eliminara a internet de la lista de gastos suntuarios y retomara la orientación del decreto 825. El movimiento Internet Prioritaria se convirtió en el primer caso documentado en Venezuela de una campaña de activismo en la web para poner un asunto en la agenda de los medios masivos, generar opinión pública e intentar incidir en una política pública (Briceño et al, 2010). La campaña web de Internet Prioritaria utilizó blogs, Facebook y Twitter. El tema de la importancia de internet para el desarrollo llegó a posicionarse en los medios masivos como consecuencia de la campaña y se obtuvieron reuniones con altos funcionarios del Ejecutivo Nacional, aunque no se logró que se revirtiera el Decreto 6.649 (Puyosa, 2015a).

Un evento importante en la disputa contenciosa de los espacios de la web ocurre el 1 de agosto de 2009, cuando se produce en Twitter una protesta con la etiqueta \#FreeMediaVE. La protesta ocurre en reacción contra la salida del aire de 34 emisoras de radio, ocurrida el 31 de julio de 2009, por disposición de CONATEL, entonces encabezado por Diosdado Cabello. \#FreeMediaVE llegó a ubicarse en el top 10 de los trending topics mundiales de Twitter. El hecho fue importante porque a partir de ese momento los periodistas y los opinadores en me - 
dios masivos venezolanos comenzaron a usar intensivamente esta plataforma de información (Puyosa, 2015a). En ese momento, la penetración de internet en Venezuela alcanzaba $31 \%$ (CONATEL, 2013); lo que implica el paso del rango de baja penetración al rango de penetración media.

A partir de ese caso, el gobierno opta por disputar estos espacios de contestación, cuya importancia en el debate político se avizoraba como creciente. No se puede descartar que la atención que el gobierno le dio al caso de \#FreeMediaVE estuviera condicionada por la repercusión global que había tenido el uso de Twitter en junio 2009, cuando la etiqueta \#IranElection sirvió para aglutinar las protestas bajo alegato de fraude en la reelección Mahmoud Ahmadinejad, aliado estratégico del chavismo. El hecho es que el gobierno comienza a utilizar sistemáticamente la web social a partir de 2010. En mayo, se crea la Misión@Chavezcandanga que incorporó a 200 funcionarios para procesar solicitudes de ayuda y denuncias recibidas por la cuenta Twitter del presidente Chávez.

En diciembre 2010, la bancada oficialista en la Asamblea Nacional ${ }^{2}$ aprobó las reformas de la Ley de Telecomunicaciones y de la Ley de Responsabilidad Social de Radio, Televisión y Medios Electrónicos que introducían la obligación a los administradores de sitios web de monitorear a los usuarios y reportar al Estado esta información. Algunos mecanismos de censura previa a contenidos políticos que se proponían no fueron aprobados en los textos definitivos de estas leyes, en buena medida por la acción de organizaciones de defensa de la libertad de expresión, tales como Espacio Público y la Coalición ProAcceso. No obstante, se aprobaron una serie de regulaciones sobre los proveedores de acceso a internet, consistentes con políticas de control de segunda generación (Drezner, 2009; Kerr, 2014).

La Ley de Responsabilidad Social de Radio, Televisión y Medios Electrónicos (RESORTE-ME) establece regulaciones de contenido para los medios digitales y para los ISPs. El artículo 27 señala que está prohibido difundir por medios electrónicos contenidos que:

1. Inciten o promuevan el odio y la intolerancia por razones religiosas, políticas, por diferencia de género, por racismo o xenofobia.

2. Inciten o promuevan y/o hagan apología al delito.

3. Constituyan propaganda de guerra.

4. Fomenten zozobra en la ciudadanía o alteren el orden público.

\footnotetext{
${ }^{2}$ El oficialismo tenía mayoría calificada de $2 / 3$ en esa Asamblea Nacional, que cesaba en sus funciones el 31 de diciembre. En la Asamblea que iniciaría funciones en enero 2011, sólo tendría mayoría simple.
} 
5. Desconozcan a las autoridades legítimamente constituidas.

6. Induzcan al homicidio.

7. Inciten o promuevan el incumplimiento del ordenamiento jurídico vigente.

(República Bolivariana de Venezuela, 2010).

Aunque la legislación entró en vigencia en 2011, estas disposiciones sólo se empezaron a aplicar sistemáticamente a partir de 2013. No obstante, el establecimiento de sanciones es completamente discrecional y no hay reglas claras sobre qué contenidos son objeto de sanciones, lo que, en la práctica, conllevó a la autocensura. En reacción a estas disposiciones a partir de 2011, la moderación comenzó a ser una práctica común en los foros en-línea venezolanos, estableciéndose así otro mecanismo de control de segunda generación en el cual son los medios digitales y los proveedores de servicios de internet los que censuran contenidos, sin que el gobierno se involucre directamente.

En agosto de 2011, más de 20 cuentas de periodistas, activistas de DDHH, escritores, infoactivistas, analistas políticos, economistas y dirigentes políticos de la Unidad Democrática fueron usurpadas mediante crackeo de sus contraseñas (Freedom House, 2012). Miembros de un grupo pro-gubernamental denominado N33 aseguraron vía Twitter y lo ratificaron a través del programa "La Hojilla" (transmitido por el canal del Estado, Venezolana de Televisión) que ellos se habían apropiado de esas cuentas. (La Hojilla- Venezolana de Televisión, Septiembre 2011).

Paralelamente, desde 2012, se han registrado varios casos de publicación por parte de funcionarios del gobierno o de activistas pro-gubernamentales de comunicaciones privadas de opositores, a pesar de que la privacidad de las comunicaciones está garantizada en la Constitución (Freedom House, 2013; Freedom House, 2015). En programas de opinión transmitidos por el canal de TV gubernamental, Venezolana de Televisión, se han presentado imágenes de correos electrónicos, grabaciones de conversaciones telefónicas e, incluso, grabaciones de conversaciones personales sostenidas en las casas de dirigentes de oposición. Este tipo de prácticas basadas en el uso de información publicada en-línea para hostigar o acusar legalmente a activistas políticos se relaciona con el "patriotic hacking" y es otra modalidad de control político de internet de segunda generación (Deibert \& Rohozinski, 2010; Drezner 2010; Kerr, 2014).

No obstante, la desinversión en el desarrollo de infraestructura pareciera ser el eje central de la política de control de internet del Estado venezolano entre 2011 y 2013. En 2011, la pe- 
netración de internet alcanzó 40\% (CONATEL, 2013), pero allí se detiene la curva de crecimiento sostenido que se había iniciado en 2003 y empieza un período de estancamiento. Esto coincide con la observación de que en los países con regímenes autoritarios se tiende a limitar el desarrollo de infraestructura de acceso a internet, lo que puede considerarse un mecanismo de control de internet de segunda generación (Drezner, 2009; Deibert \& Rohozinski, 2010; Kerr, 2014). En los reportes oficiales de CONATEL se observa un declive en la penetración de internet en las entidades rurales y fronterizas (CONATEL, 2013; 2014; 2015a). Simultáneamente, se observa el deterioro de la infraestructura, con la consiguiente disminución de la velocidad de las conexiones. En 2012 se registra una velocidad de descarga promedio de 1,95 Mbps frente a un promedio de 4,13 Mbps para toda Latinoamérica, sexta velocidad en Suramérica; mientras que en 2014 se registra una velocidad de descarga promedio de 2,31 Mbps, frente a un promedio de 7,26 Mbps para Latinoamérica, décima y última de Suramérica (Jordán, Galperin \& Peres, 2013; Rojas \& Poveda, 2015). Estas tendencias configuran un patrón de negación de acceso a internet, que resulta evidente desde 2011, año a partir del cual se observa el retraso en la ejecución del Octavo Proyecto de Servicio Universal o Red Nacional de Transporte, que tiene como objetivo la construcción de infraestructura de fibra óptica para proporcionar servicios de telecomunicaciones en 18 de las 24 entidades del país, que debió culminarse en 2012 (CAF, 2013; CONATEL, 2015b), más a la fecha sigue reportándose como en proceso (CONATEL, 2015c).

\section{3-2015:}

\section{El control de internet en un régimen híbrido con tendencias autocráticas}

A partir de 2013, el gradual movimiento del chavismo desde su inicial modelo de democracia delegativa alcanza características cada vez más autocráticas, aunque se mantengan algunas formalidades e instituciones democráticas como las elecciones competidas, que permiten que se le siga considerando un régimen híbrido (Corrales \& Hidalgo, 2013; Chaguaceda \& Puerta, 2015). En este período se institucionalizan políticas públicas de control de internet que rompen definitivamente con el modelo democratizador del Decreto 825. Se observan formas cada vez más sofisticadas de controles de segunda generación y la acentuación de la disputa contenciosa en la web. Simultáneamente se observa un conspicuo uso de mecanismos de vigi lancia y la judicialización como respuesta a la expresión de disenso político en la web. 
Asimismo se hacen evidentes las prácticas de filtrado, bloqueo y censura de contenidos en la web por parte de gobierno de Venezuela, mecanismos de denegación de acceso de primera generación que no habían sido utilizados extensivamente por el gobierno chavista durante las fases anteriores.

Los cambios en la política para internet del Estado venezolano alcanzan expresión institu cional en el II Plan Socialista de Desarrollo Económico y Social de la Nación 2013-2019, conocido como Plan de la Patria. Entre las secciones del Plan de la Patria, la más importante para nuestro objeto de estudio es el Gran Objetivo Histórico $\mathrm{N}^{\circ} 4$. De allí se deriva el Objetivo Nacional

4.4. "Desmontar el sistema neocolonial de dominación imperial" que da paso al Objetivo Estratégico

4.4.2. "Reducir el relacionamiento económico y tecnológico con los centros imperiales de dominación a niveles que no comprometan la independencia nacional”. Dentro de este objetivo estratégico se derivan los Objetivos Generales:

4.4.2.3. "Llevar a niveles no vitales la conexión de Venezuela con las redes de comunicación e información dominadas por las potencias neocoloniales".

4.4.2.4. "Eliminar la dependencia de sectores estratégicos para el desarrollo nacional de redes de comunicación e información controladas por las potencias neocoloniales".

Obviando la retórica grandilocuente del Plan de la Patria, es necesario destacar que el Plan de la Nación 2013-2019 tiene como objetivo fundamental "Garantizar la continuidad y consolidación de la Revolución Bolivariana”. Esto es un proyecto político-partidista elevado a razón de Estado. Igualmente, se eleva a política de Estado el impulso al "socialismo bolivariano" como modelo político y económico para Venezuela y para los países aliados en Latinoamérica. Asi mismo, el Plan de la Patria recalca el rol protagónico de la Fuerza Armada Bolivariana en este modelo político y propone la unidad cívico-militar como base de la seguridad del Estado venezolano, a lo cual se ata la continuidad de la Revolución Bolivariana. Finalmente, el Plan de la Patria se propone el fortalecimiento del Poder Popular y el modelo de organización políticoterritorial de las comunas, ambas figuras extra-constitucionales que modifican la estructura del Estado venezolano y de su sistema político. Además a este proyecto político nacional se le atribuye un rol en la geopolítica internacional que parece atado a la confluencia de intereses con otros países, entre los cuales se destaca a China, Rusia, Irán, Brasil y Belarus. Rusia, en particular, es el aliado más influyente en términos de políticas de control de internet. 
Este es el contexto que enmarca las políticas sobre tecnologías de información y comuni cación, y específicamente sobre uso de internet, en el período 2013-2015. En este lapso, efectivamente la conectividad a internet en Venezuela ha alcanzado niveles mínimos. Asimismo, en este período han aumentado las regulaciones sobre contenidos y penalizaciones a usuarios de internet en función de los objetivos de consolidación de la Revolución Bolivariana.

Desde junio 2014, CONATEL estableció como práctica rutinaria el bloqueo de páginas web que publicaban tasas de cambio del mercado paralelo. El 18 de noviembre, CONATEL solicitó a Twitter el bloqueo de las cuentas en esa plataforma de los servicios informativos que reportaban tasas de cambio diferentes a las establecidas por el control gubernamental. Adicionalmente, CANTV estableció medidas para el bloqueo de tweets que contuvieran enlaces que usaran el acortador Bit.ly, simplemente porque el servicio web de información sobre el mercado negro más popular usaba ese acortador de URLs (Freedom House, 2014).

Existe evidencia de que durante los años 2012 y 2013 el gobierno de Venezuela utilizó Blue Coat PacketShaper, un paquete para filtrado, censura y vigilancia en internet (The Citizen Lab, 2013). También habría que tomar nota de que en 2013, la empresa italiana de ciberespionaje Hacking Team recibió solicitudes de demostraciones de sus servicios de parte de entes venezolanos, como el Ministerio de Política Interior, Justicia y Paz. Asimismo, en 2013, fue creado el CESPPA, una oficina nacional de seguridad que reporta directamente a la Presidencia de la República y tiene facultades para interceptar comunicaciones personales en la web sin orden judicial. El CESPPA tiene atribuciones para decidir discrecionalmente que las comunicaciones de una persona pudiesen afectar los intereses estratégicos del Estado, y si ese es el caso interceptar dichas comunicaciones. ${ }^{3}$

El 14 de abril de 2013, día de elecciones presidenciales, CANTV, la empresa de telecomunicaciones gestionada por el gobierno — que proporcionaba acceso a internet para cerca de $80 \%$ de los suscriptores venezolanos- paralizó sus servicios por aproximadamente $35 \mathrm{mi}$ nutos, dejando a la mayoría de los venezolanos desconectados de internet precisamente en la hora en que debían comenzar a cerrarse los centros de votación (Freedom House, 2014). Incluso después de que CANTV re-estableció sus servicios de acceso a internet, el sitio web del Consejo Nacional Electoral (CNE) se mantuvo fuera de línea durante varias horas. En la mañana del 15 de abril, el sitio web del CNE volvió a estar disponible para quienes se conectaban desde Venezuela, pero el acceso permaneció bloqueado para conexiones desde otros países. El

\footnotetext{
${ }^{3}$ En 2010, se había creado una agencia similar, el CESNA, pero el Tribunal Supremo de Justicia dictaminó que violaba derechos constitucionales y tuvo que ser eliminada.
} 
incidente fue particularmente serio debido a que el presidente provisional Nicolás Maduro fue declarado ganador de la elección con un margen de apenas $1 \%$ de los votos a favor. El vicepresidente (y yerno del fallecido presidente Hugo Chávez) Jorge Arreaza reconoció en declaraciones a los medios que él personalmente había ordenado que se quitara el acceso a in ternet (Alba Ciudad (Abril 14, 2013).

$\mathrm{Ni}$ las masivas manifestaciones estudiantiles de protesta contra del gobierno de febreromayo de 2014 ni la represión gubernamental fueron cubiertas por la televisión nacional; y su cobertura por radio y medios impresos fue parcial y minimizada (Chirinos, 2014). CONATEL instruyó a los proveedores de TV por suscripción para que sacaran del aire la señal del canal NTN24 (basado en Colombia), que estaba dando extensa cobertura a las protestas en Venezuela. Los ciudadanos con acceso a internet se volcaron a Twitter, Facebook y los sitios web de medios internacionales que permitían obtener alguna información sobre las manifestaciones que estaban ocurriendo en las calles de Venezuela (Delgado \& Arenas, 2014; Puyosa, 2014). En un cambio claro de las políticas de control de internet hacia un modelo más represivo, a partir de febrero de 2014, se adoptan medidas de primera generación de filtrado y bloqueo. Cerca de 500 sitios web fueron bloqueados para el acceso desde Venezuela durante el mes de febrero 2014 (Espacio Público, 2015), en respuesta a la migración de las audiencias interesadas en las noticias de los medios tradicionales a la web. En el estado Táchira, donde se habían iniciado las protestas el 4 de febrero, el acceso a internet fue completamente cortado durante más de 3 días, mientras la Guardia Nacional ${ }^{4}$ tomaba las calles para reprimir las manifestaciones (Freedom House, 2015).

En la plataforma Herdict — basada en el Berkman Center de Harvard University — se re portaron 1.949 intentos de acceso a sitios web que no pudieron resolverse con éxito desde el ISP gubernamental CANTV o el ISP privado Inter, durante el período del 10 de Febrero al 10 de Abril de 2014 (Herdict, Febrero-Abril 2014). Los sitios web que acumularon más reportes de inaccesibilidad fueron www.ntn24.com, pastebin.com, www.diariodecuba.com y dollar.nu (Espacio Público, 2015). También existen reportes técnicos que indican que entre mediados de febrero y finales de marzo 2014, CANTV y Movilnet pusieron un límite de 1Mgbps durante las tardes y noches en que se desarrollaban manifestaciones de protesta. Existen denuncias de que entre febrero y marzo de 2014, la empresa gubernamental proveedora de acceso a internet, CANTV, manipuló la velocidad del tráfico de datos hacia los servidores de Twitter y YouTube (Freedom House, 2015). La ralentización de las conexiones hacia estos ser-

\footnotetext{
${ }^{4}$ Cuerpo de la Fuerza Armada Bolivariana al cual se le asignan funciones policiales.
} 
vicios de web social habría tenido como objetivo obstaculizar la difusión de imágenes de las manifestaciones de protesta y de las acciones represivas por parte de policías, militares y grupos parapoliciales. Vemos así que durante el ciclo de protestas de 2014 se observó un amplio repertorio de prácticas de control de segunda generación, que incluyó corte total de acceso a internet en regiones en donde se producen protestas, bloqueo just-on-time de páginas web o aplicaciones móviles, y ralentización de las conexiones a internet.

Con posterioridad al ciclo de protestas que culmina en mayo 2014, se avanza en el establecimiento de políticas de ciberdefensa sustentadas en nociones de seguridad nacional. El 12 de agosto de 2014 se crea la Dirección Conjunta de Seguridad Informática (DICOCEI) de la Fuerza Armada Bolivariana (FANB) que incluía una División de Redes Sociales (Gaceta Oficial, 12 de agosto de 2014). Posteriormente, se creó la Dirección Conjunta de Ciberdefensa de la Fuerza Armada Bolivariana (FANB) (Gaceta Oficial, 7 de Mayo de 2015). Estos organismos tienen facultades para ejecutar acciones de vigilancia masiva en la web, bajo el argumento de riesgos para la seguridad nacional.

Desde 2014, se han registrado casos de persecución y encarcelamiento de usuarios de la web, específicamente usuarios de Twitter. Entre finales de agosto y principios de octubre 2014, 7 usuarios de Twitter fueron detenidos por la policía política SEBIN por publicar tweets alusivos al asesinato del diputado del PSUV, Robert Serra. Hasta noviembre de 2015, 6 de estos ciudadanos permanecían en prisión sin que se les haya sometido a juicio (Freedom House, 2015).

Hasta la fecha, el caso más conspicuo de judicialización del uso de la web es el juicio contra el dirigente político Leopoldo López, quien se encuentra en una cárcel militar desde fe brero de 2014 y fue condenado en septiembre de 2015 a más de 13 años de prisión por los delitos de determinador en el delito de incendio intencional, instigación pública, daños a la propiedad pública y asociación para delinquir. El primer elemento que se presenta para la acusación en el expediente es un tweet y el segundo un video en YouTube. El video al que se refiere la acusación es el discurso de Leopoldo López para el 23 de enero (fecha en que se conmemora el derrocamiento de la dictadura del general Marcos Pérez Jiménez) (López, Leopoldo, Enero 22 2015). El video fue difundido a partir de un tweet emitido desde la cuenta @leopoldolopez y en la acusación se destaca la frase: "TENEMOS QUE SALIR A CON QUISTAR LA DEMOCRACIA", a la cual se le atribuye contenido violento y motivaciones desestabilizadoras (Ministerio Público, 2014 expediente № C-16-17936-2014). El testigo clave de la acusación fue una lingüista que analizó el discurso de @leopoldolopez para con- 
cluir se usó esa cuenta en Twitter para convocar subliminalmente manifestaciones en contra del gobierno.

Paralelamente, se ha ido ampliando el alcance de la línea de hegemonía comunicacional de los medios masivos a la web social. En este sentido, la política para internet incluye el uso de bots y trolls para sumergir las expresiones de protesta y para configurar la agenda de debate público en la web (Thomas, Grier \& Paxson, 2012; Verkamp \& Gupta, 2014). El SIBCI (Sis tema Bolivariano de Comunicación e Información), a través de la actividad de sus funcionarios y sus bots, posiciona diariamente etiquetas que llegan artificialmente a los trending topics sumergiendo los temas que realmente están debatiendo los usuarios de Twitter en Venezuela. Se utilizan mecanismos de social spam y trolling, que incluyen la fabricación de pseudo-noticias a partir de tweets polémicos de funcionarios públicos y de los trending topics impulsados desde el SIBCI. El hecho de que muchos funcionarios públicos no declaren a los periodistas, sino que los dirijan a seguir su usuario en Twitter para recibir información, ayuda a consolidar ese canal unidireccional de comunicación de gobierno. Esta táctica facilita "pautar" a los medios, dirigiéndolos a hacerle seguimiento a pseudo-noticias, que de otra manera no tendrían ninguna incidencia en la opinión pública.

Finalmente, en 2015, el gobierno de Venezuela ha comenzado a trabajar en una nueva línea estratégica para el cerco y la ocupación de los espacios de contestación en la web, que se deriva del Plan de la Patria. Se trata de la creación de la Red Patria, que había sido anunciada en diciembre de 2011 por Hugo Chávez. El proyecto comenzó a desarrollarse en octubre de 2012, en concordancia con el Plan de la Patria 2013-2019, mencionado más arriba. Responde a los objetivos:

1.1.5.9. "Conformar un sistema de medios que contribuya a la organización sectorial para la defensa integral de la Patria, con énfasis en la consolidación de nuevos medios y formas de producir contenidos en la frontera con relevancia de los valores patrióticos y socialistas".

4.4.2.4. "Eliminar la dependencia de sectores estratégicos para el desarrollo nacional de redes de comunicación e información controladas por las potencias neocoloniales".

Desde el punto de vista de avanzar en la hegemonía comunicacional del Estado chavista, re sulta necesario establecer espacios en internet controlados por el Estado que se orienten a sustituir a los espacios de la web social que son populares en la actualidad y que no son controlados por el gobierno. El gobierno ha insistido en cuestionar el uso de la web social, por tratarse de servicios proporcionados por corporaciones multinacionales; más en la práctica in- 
vierte en garantizar la presencia de los entes públicos directamente en Twitter y vía publicidad pagada en Google Ad Sense. El desarrollo de la Red de la Patria tiene el propósito de facilitar a la población plataformas de web social que estén bajo el control del Estado venezolano y sirvan para apoyar el fortalecimiento del poder comunal y el avance en el proyecto político bolivariano.

\section{Conclusiones}

En el estudio del caso Venezuela 2007-2015, se observa como la política pública para internet se fue modificando en la medida en que el régimen político pasó de ser una democracia delegativa a ser un régimen con predominio de rasgos autoritarios. A partir de 2007, el incremento de los debates críticos al gobierno chavista y al proceso bolivariano en el espacio de la web habría llevado a la élite en el poder a valorar la necesidad de establecer controles políticos en internet.

De acuerdo con las proposiciones del "dilema del dictador", en caso de regímenes híbridos como el venezolano tienden a usarse preferentemente formas sofisticadas y selectivas de control político de internet de segunda generación, en la medida en que aumenta la penetración de internet y su uso para activismo político. Efectivamente, observamos que hasta 2007, el gobierno venezolano no interfirió significativamente con la libre expresión política en internet. A partir de ese año comienza a establecer políticas públicas que frenaban el acceso a internet, a partir principalmente de la desinversión en desarrollo de la infraestructura, la creación de barreras para la inversión privada (incluyendo la estatización del principal ISP) y el establecimiento de barreras para su uso en instituciones públicas. Este tipo de medidas tienen la ventaja de no afectar la reputación internacional del país, porque no son consideradas limitaciones a derechos civiles y políticos, sino que pasan como efectos colaterales del subdesarrollo. Por el contrario, medidas como el punto único de acceso nacional a internet que habría facilitado el bloqueo y filtrado de contenidos, fueron consideradas y desechadas en 2008, probablemente por los altos costos en términos de reputación que tienen estos controles de primera generación.

Es a partir de 2010, cuando el gobierno de Venezuela comienza a utilizar un amplio repertorio de controles de segunda generación. Ocurre justamente cuando la penetración de internet en el país alcanza un tercio de la población y el debate político en la web comienza a tener incidencia en la formación de opinión pública. A partir de 2010, el régimen chavista pone en 
práctica controles sobre los proveedores de servicios de internet, grupos de "ciberactivistas" pagados para interferir en debates en la web sobre asuntos políticos (e.g. Misión Chávezcandanga, \#LaTropa y los bots del SIBCI), requerimiento informales de remoción de contenidos a ISPs y a administradores de sitios web, bloqueo selectivo de contenidos con justificación legal (eg. informaciones sobre mercado cambiario paralelo), uso de información publicada en-línea para hostigar o acusar legalmente a activistas políticos, ciberataques perpetrados por crackers contra activistas opositores o "patriotic hacking".

A partir de 2013, con el desplazamiento hacia un régimen cada vez más autoritario y con menor legitimidad, se usan mecanismos de control más agresivos como vigilancia masiva por inspección de paquetes de datos, ralentización o corte total de acceso a internet en momentos de alta conflictividad política, bloqueo just-on-time de páginas web o aplicaciones móviles. Ese año se crea la primera agencia gubernamental con funciones de control político de internet, el CESPPA.

Como reacción al ciclo de protestas de 2014, se acentúa el uso de estos mecanismos de control de segunda generación. Se observa el uso sistemático de bloqueo just-on-time de páginas web o aplicaciones móviles, así como la ralentización de las conexiones a internet. También se registran cortes totales de acceso a internet en regiones en donde se producen protestas. Asimismo, es en 2014 cuando se observa por primera vez en Venezuela el bloqueo de contenidos por razones estrictamente políticas, sin ninguna justificación legal, como reacción al movimiento masivo de protesta. Luego de finalizado el ciclo de protestas, se adoptan controles no técnicos como los reportes policiales rutinarios de la actividad ciudadana en internet y la prisión para usuarios por expresiones políticas en Twitter.

Asimismo, en 2015 se empiezan crear órganos militares con funciones de control político de internet, legitimadas con un discurso de seguridad y defensa. Lo que pareciera indicar que el régimen chavista está optando por mecanismos de control de internet cada vez más generalizados, sin que ya se impongan los costos de reputación internacional y legitimidad interna frente a los costos de mantener la estabilidad en el poder en un contexto de creciente conflicti vidad política que se expresa principalmente a través de la web. 


\section{Referencias}

Alvarez, A. (2011). Countries at the Crossroads 2011: Venezuela. Freedom House.

Benítez, S. y Pietrosemoli, E. (2012). Caso Venezuela. En Asociación para el Progreso de las Comunicaciones. Uso y regulación del espectro en América Latina. Estudios de caso de Argentina, Brasil, Ecuador, Perú y Venezuela. Quito, Ecuador: Ediciones CIESPAL.

Bisbal, M., Oropeza, A., Hernández, G., Urribarri, R., Cañizalez, A, Correa, C., Abreu, I. y R. QuiÑonez (2009). Hegemonía y control comunicacional. Caracas, Venezuela: Editorial Alfa/UCAB.

Briceño, Y. (2009). Venezuela en siglo de cambios: Nueve años de vaivenes en las comunicaciones. Anuario Electrónico de Estudios en Comunicación Social "Disertaciones", 2 (1), Artículo 5.

Briceño, Y.; Núñez, L.; Pisanty, A.; Puyosa, I.; Urribarrí, R. y R. Torrens (2010). Políticas y demandas civiles en la sociedad interconectada. Una revisión de movimientos en defensa de Internet en México y Venezuela. En Said, Elías (ed.) TIC, comunicación y periodismo digital. Reflexiones de América Latina y Europa. Barranquilla, Colombia: Ediciones UnNorte.

CAÑIzalez, A. (2012) Chávez: La presidencia mediática. Caracas, Venezuela: Editorial Alfa.

Chaguaceda, A. y M. I. Puerta (2015). “Quo vadis Venezuela”: De la democracia delegativa al autoritarismo del siglo XXI. Revista Mexicana de Análisis Político y Administración Pública, 4(1), 175-202.

Chirinos, M. (2014). Represión y censura. Revista Comunicación. n. 165. Caracas, Venezuela: Centro Gumilla.

Corrales, J. y M, Hidalgo. (2013). El régimen híbrido de Hugo Chávez en transición (20092013). Desafíos, 25(1), 45-84.

Crete-Nishinata, M., Deibert, R., y Senft, A. (2013). Not by technical means alone: the multidisciplinary challenge of studying information controls. Internet Computing. IEEE, 17(3), 34-41.

DeIBERT, R., y Rohozinski, R. (2010). Liberation vs. control: The future of cyberspace. Journal of Democracy, 21(4), 43-57.

Delgado-Flores, C. y G. Arenas. (2014). \#12F la rebelión digital. Revista Comunicación. Segundo trimestre 2014. Caracas, Venezuela: Centro Gumilla. 
Drezner, D. W. (2009). Weighing the scales: The Internet's effect on state-society relations. Brown Journal of World Affairs, 16, 31.

Howard, P. N., Agarwal, S. D., y Hussain, M. M. (2011). When do states disconnect their digital networks? Regime responses to the political uses of social media. The Communication Review, 14(3), 216-232.

Jordán, V., Galperin, H. y Peres, W. (2013). Banda ancha en América Latina: más allá de la conectividad. Santiago de Chile, Chile: CEPAL.

KerR, J. (2014). The Digital Dictator's Dilemma: Internet Regulation and Political Control in Non-Democratic States, presentado en Social Science Seminar Series, The Center for International Security and Cooperation (CISAC), Stanford University, October 16, 2014. Retrieved from:

$<$ http://fsi.stanford.edu/events/digital-dictator\%E2\%80\%99s-dilemma-internet-regulationand-political-control-non-democratic-states-0>

Morozov, E. (2012). The net delusion: The dark side of Internet freedom. Nueva York, US: Public Affairs.

Murdoch, S. J., y Roberts, H. (2013). Introduction to: Internet Censorship and Control. Disponible en SSRN 2268587.

O'Donnell, G. A. (2011). Democracia delegativa. En O'Donnell, G. A., Lazzetta, O., \& Quiroga, H. Democracia delegativa. Buenos Aires, Argentina: Prometeo libros.

Pearce, K. E., y Kendzior, S. (2012). Networked authoritarianism and social media in Azerbaijan. Journal of Communication, 62(2), 283-298.

Puyosa, I. (2012). Conectados versus Mediáticos. ¿Politizados o des-politizados? Anuario de Estudios en Comunicación Social "Disertaciones". Vol 5, No 1 (Julio 2012).

Puyosa. I. (2014). El contagio de ideas políticas, la identidad colectiva y los movimientos sociales en redes. Con un punto aparte para el \#Movimiento12F. Revista Comunicación. Segundo trimestre 2014.

Puyosa, I. (2015a). En-línea de investigación: Investigación sobre comunicación e internet en Venezuela (2007-2013). En Bisbal, M. \& María Aguirre, J.M. Encrucijadas de la Comunicación en Venezuela. Caracas: Centro Gumilla / Bid \& Co, Editor.

Puyosa. I. (2015b). Los movimientos sociales en red: Del arranque emocional a la propagación de ideas de cambio político. Revista Chasqui. № 128.

Rojas, E. y Poveda, L. (2015). Estado de la banda ancha en América Latina y el Caribe 2015. Santiago de Chile: CEPAL. 
SABATIER, P.A. (1993). Policy change over a decade or more. In Sabatier, P. \& Jenkins-Smith, H. Policy Change and Learning: An Advocacy Coalition Approach. Boulder, CO: Westview Press.

Sabatier, P.A. y Jenkins-Smith, H. (1993). The Advocacy Coalition Framework. Assessment, Revisions, and Implications for Scholars and Practitioners. In Sabatier, P. \& Jenkins-Smith, H. Policy Change and Learning: An Advocacy Coalition Approach. Boulder, CO: Westview Press.

Sabatier, P. A., y Weible, C. M. (2007). The Advocacy Coalition Framework: Innovations and Clarifications, Theories of Policy Process. Boulder, CO: Westview Press.

Thomas, K., Grier, C., y Paxson, V. (2012). Adapting social spam infrastructure for political censorship. En Proceedings of the 5th USENIX conference on Large-Scale Exploits and Emergent Threats (pp. 13-13). USENIX Association.

Verkamp, J. P., y Gupta, M. (2014). Five incidents, one theme: Twitter spam as a weapon to drown voices of protest. En Third USENIX Workshop on Free and Open Communications on the Internet. Recuperado de:

<https://www. usenix. org/conference/foci13/technical-sessions/papers/verkamp>

URribarri, R. (2011). Redes sociales y medios digitales: ¿Alternativa comunicacional en Venezuela? Revista Comunicación. Cuatro trimestre 2011.

Urribarri, R. (2013). De la Sociedad del Conocimiento al Socialismo del Siglo XXI. Ponencia presentada en el IV Congreso de Investigadores Venezolanos de la Comunicación. Barquisimeto 29 y 30 de abril de 2013.

Weible, C. M., y Nohrstedt, D. (2012). Coalitions, learning and policy change. Routledge handbook of public policy, 125-137.

\section{Documentos oficiales}

CONATEL (2000). Plan Nacional de Telecomunicaciones. Recuperado de: $<$ http://Fisis.faces.ula.ve/FCOMPUTACION/Varios/Plan_Nacional_Telecomunicac.doc>

CONATEL. (2013) Servicio de Internet. Indicadores. 1998-2012. Recuperado de: $<$ http://www.conatel.gob.ve/index.php/principal/indicadoresanuales>

CONATEL. (2014) Indicadores anuales 2013. Recuperado de: $<$ http://www.conatel.gob.ve/files/Indicadores/indicadores_anuales2013/PRESENTACION_A NUAL2013defi.pdf>

CONATEL. (2015a) Indicadores anuales 2014. Recuperado de: 
$<$ http://www.conatel.gob.ve/files/Indicadores/indicadores_2014/CIFRAS_I2014_COMPLETO.pdf>

CONATEL. (2015b). Red Nacional de Transporte. Recuperado de:

$<$ http://www.conatel.gob.ve/octavo-proyecto/>

CONATEL. (2015c). Medios Comunitarios. Recuperado de:

$<$ http://www.conatel.gob.ve/noveno-proyecto/>

Gaceta Oficial (12 de agosto de 2014). № 40.557.

Gaceta Oficial (7 de Mayo de 2015). № 40.655.

Ministerio Público, 2014. Fiscalía Segunda, Cuadragésima Primera y Cuadragésima Quinta A Nivel Nacional con Competencia Plena Expediente № C-16-17936-2014. Recuperado de: <http://cdn.eluniversal.com/2014/06/02/ACUSACION_LEOPOLDO.pdf>

MPPTI (2007). Plan Nacional de Telecomunicaciones 2007-2013. Recuperado de: $<$ http:///www.cnti.gob.ve.>

Presidencia de la República Bolivariana de Venezuela (2000). Decreto 825. Gaceta Oficial N 36955. 10 de mayo de 2000. Recuperdo de:

$<$ http://www.cecalc.ula.ve/internetprioritaria/documentos/decreto_825.pdf>

Presidencia de la República Bolivariana de Venezuela. (2007). Proyecto Nacional Simón Bolívar. Primer Plan Socialista de la Nación: 2007-2013. Recuperado de: $<$ http://repositorio.mpd.gob.ve/20/>

Presidencia de la República Bolivariana de Venezuela (2009). Decreto 6.649. Instructivo Presidencial para la Eliminación del Gasto Suntuario o Superfluo en el Sector Público Nacional. Gaceta Oficial № 39.146, 25 de marzo de 2009. Recuperado de: <http://www.cecalc.ula.ve/internetprioritaria/documentos/decreto6649.pdf>

República Bolivariana de Venezuela (2010). Ley de Responsabilidad Social en Radio, Televisión y Medios Electrónicos. Recuperado de:

$<$ http://www.leyresorte.gob.ve/wp-content/uploads/2012/07/Ley-de-Responsabilidad-Socialen-Radio-Television-y-Medios-Electr\%C3\%B3nicos.pdf>

\section{Informes}

Corporación Andina de Fomento (2013). Sector TIC: Venezuela (Sector Tic). Caracas: CAF. Recuperado de:

<http://scioteca.caf.com/handle/123456789/590> 
Espacio Público (2015) Situación general de la libertad de expresión e información en Venezuela (enero-diciembre 2014). Recuperado de: $<$ http://www.derechos.org.ve/pw/wp-content/uploads/253194052-INFORME-SITUACIO-NDE-LA-LIBERTAD-DE-EXPRESION-E-INFORMACIO-N-EN-VENEZUELA-2014.pdf>

Freedom House (2012) Freedom on the Net. A Global Assessment of Internet and Digital Media Freedom.

Freedom House (2013) Freedom on the Net. A Global Assessment of Internet and Digital Media Freedom.

Freedom House (2014) Freedom on the Net. A Global Assessment of Internet and Digital Media Freedom.

Freedom House (2015) Freedom on the Net. A Global Assessment of Internet and Digital Media Freedom.

The Citizen Lab (2013). Planet Blue Coat: Mapping Global Censorship and Surveillance Tools. Toronto: Munk School of Global Affairs.

\section{Web}

Alba Ciudad (Abril 14, 2013) (VIDEO) Jorge Arreaza: suspensión momentánea de Internet se hizo para proteger a la página web del CNE de ataques. Recuperado de:

$<$ http://albaciudad.org/wp/index.php/2013/04/video-jorge-arreaza-suspension-momentaneade-internet-se-hizo-para-proteger-a-la-pagina-web-del-cne-de-ataques/>

Herdict. ( February 10 to April 10, 2014). Venezuela Report. Berckman Center. Harvad University. Recuperado de:

$<$ https://www.herdict.org/explore/time; jsessionid=E7176C6A1721D2E5F250DA41C3A34C $79 \# \mathrm{fc}=\mathrm{VE} \& \mathrm{fed}=04 / 10 / 2014 \& \mathrm{fl}=\& \mathrm{fs}=\& \mathrm{fsd}=02 / 10 / 2014 \& \mathrm{ft}=$ inaccessible Acceso: Julio 17, 2014>

La Hojilla - Venezolana de Televisión (Septiembre 2011). Recuperado de:

$<\mathrm{http}: / /$ www.youtube.com/watch?v=MHRFuCItKLA>

López, Leopoldo (Enero 22, 2015). Palabras de Leopoldo 23E 2014, hoy más vigentes que nunca. Recuperado de:

$<$ https://www.youtube.com/watch?v=8_IotCyLiQE\&feature=youtu.be> 